\title{
La grabadora de voz del celular, como herramienta para incentivar la producción oral en inglés
}

\section{The cell phone voice recorder, as a tool to encourage oral production in English}

\author{
RODRÍGUEZ TAMAYO, Ilba Y. ${ }^{1}$ \\ MARTÍN, Yenny A. ${ }^{2}$ \\ NÁJAR SÁNCHEZ, Olga ${ }^{3}$
}

\begin{abstract}
Resumen
Esta investigación propone una secuencia didáctica para analizar la incidencia de la grabadora de voz del celular, en la producción oral de un grupo de estudiantes. El estudio siguió un enfoque mixto, enmarcado en la metodología de la investigación acción. Como instrumentos se utilizaron un pre test, una secuencia didáctica, un post test, un cuestionario y una entrevista. La aplicación grabadora de voz facilitó la autoevaluación de los estudiantes; generó aprendizaje cooperativo y fortaleció la producción oral de los estudiantes.

Palabras clave: secuencia didáctica, producción oral, aplicación grabadora de voz, aprendizaje cooperativo
\end{abstract}

\begin{abstract}
This research proposes a didactic sequence in order to analyze how the cell-phone voice recorder affects the oral production in a group os students. It follows a mixed approach under the action research methodology. A pret-test, a didactic sequence, a post-test, a questionnaire and a survey were used as research instruments. The voice recorder enabled students'self assessment. It generated cooperative learning and strengthen their oral production.

key words: didactic sequence, oral production, Voice Recorder Application, cooperative learning.
\end{abstract}

\section{Introducción}

El aprendizaje de una lengua extranjera se ha convertido en un fenómeno cada vez más común en la sociedad moderna en la que vivimos, ya sea por intercambios económicos, sociales o culturales. Por consiguiente, el acto comunicativo social ha cobrado gran importancia en muchos contextos y más aún, en el campo educativo. Al hacer referencia al aprendizaje de una lengua extranjera, se habla del desarrollo de las cuatro habilidades

\footnotetext{
1 Profesora de la Licenciatura en Idiomas Modernos. Licenciada en Idiomas Modernos. Magister en Lingüística aplicada a la enseñanza del Inglés. Docente Escuela de Idiomas. Secretaria de Consejo Académico. Universidad Pedagógica y Tecnológica de Colombia. Investigadora. Grupo de Investigación Ambientes Virtuales Educativos AVE. Ilba.rodriguez@uptc.edu.co. https://orcid.org/0000-0002-5220-5951.

2 Docente Institución Educativa Antonio José Sandoval Gómez, Tunja, Colombia. Investigadora grupo Ambientes Virtuales Educativos AVE Uptc. Adrianamartin11@gmail.com. https://orcid.org/0000-0001-5615-1842

3 Profesora de la Licenciatura en Informática y Tecnología, Maestría en Educación y Maestría en Ambientes Educativos Mediados por TIC, programas adscritos a la Facultad Ciencias de la Educación de la Universidad Pedagógica y Tecnológica de Colombia UPTC. Coordinadora del grupo de Investigación Ambientes Virtuales Educativos AVE. Ingeniera de Sistemas Con Énfasis En Software. Especialista en auditoría de sistemas. Especialista en computación para la docencia. Magister en Tecnologías de la Información Aplicadas a La Educación. Estudiante del Doctorado Formación en la Sociedad del Conocimiento, Universidad de Salamanca, España.olga.najar@uptc.edu.co; olga.najar@usal.es. https://orcid.org/0000-0003-1286-7683.
} 
comunicativas: leer, escribir, escuchar y hablar. En el caso específico de esta investigación, se centra en la producción oral en inglés de los estudiantes de una institución educativa pública en Tunja, Colombia.

Así como la sociedad va cambiando, la tecnología, desde hace muchos años, ha venido incursionando en la vida de los seres humanos, haciendo que la comunicación sea cada vez más inmediata e instantánea, creando diversas formas de interacción social (Robayo, 2016) y nuevas redes de conocimiento. Con base en lo anterior, en este estudio, el interés se centró en analizar la incidencia del uso de la grabadora de voz del celular, en la producción oral en inglés de los estudiantes de una institución pública de Tunja. No obstante, no solo se estudió la parte lingüística y el uso de la grabadora, sino la incidencia en el aspecto humano del estudiante. Según Gutiérrez, Fernández \& Tabasso (2017) si se pretende lograr aprendizajes significativos por medio de las TIC, se hace necesario distanciarse del carácter meramente instrumental, para darle una faceta humanizadora a las TIC. En este caso, alrededor de la grabadora de voz, se produjo diversas interacciones sociales entre los estudiantes, que potencializó no solo la construcción de conocimiento en el aprendizaje de una lengua extranjera como lo es el inglés, sino que hubo el desarrollo de valores humanos como el respeto y el trabajo cooperativo, entre otros.

\section{Metodología}

Para conocer lo que revelaba el uso de la aplicación grabadora de voz del celular, como estrategia didáctica en la producción oral en inglés, en estudiantes de grado $9^{\circ}$ de una Institución Educativa pública en la ciudad de Tunja, Colombia, se siguió la metodología de la investigación acción, es decir, buscando mejorar la práctica educativa (Camacho, 2003); y desarrollando un enfoque mixto (Hernández, Fernández, \& Baptista, 2014), orientado con los objetivos específicos: reconocer la ansiedad de los estudiantes al momento de hablar en inglés; describir las implicaciones del trabajo cooperativo en la producción oral en inglés en los estudiantes de grado $9^{\circ}$; y evaluar el desempeño académico de los estudiantes en la producción oral en inglés mediado por el uso de la aplicación grabadora de voz del celular.

Esta investigación es de tipo Investigación Acción, con el propósito de transformar y cambiar social y educativamente, a través del mejoramiento permanente de la eficacia de la acción social; como lo sustenta Camacho (2003), la investigación acción pretende mejorar la práctica educativa. En palabras de Elliott (1990) la investigación - acción interpreta "lo que ocurre" desde el punto de vista de quienes actúan e interactúan en la situación problema, por ejemplo, profesores y estudiantes, los hechos se interpretan como acciones y transacciones humanas" (p. 5). En el caso concreto de esta investigación, mediante la intervención pedagógica, se analizó la incidencia del uso de la grabadora de voz del celular, en el desarrollo de la producción oral de los estudiantes de una Institución Educativa pública de la ciudad de Tunja.

Esta investigación es de enfoque mixto, ya que como lo sustenta Lieber y Weisner (Hernández et al., 2014) los métodos mixtos "capitalizan" la naturaleza complementaria de las aproximaciones cuantitativa y cualitativa, es decir, los fenómenos se representan mediante el uso de variables numéricas y gráficas y, a su vez, a través de textos, narrativas, etc. En palabras de Todd, Nerlich y McKeown (Hernández et al., 2014) "el enfoque mixto permite una mejor "exploración y explotación" de los datos recolectados" (p. 537).

La población seleccionada para esta investigación fueron los estudiantes de grado $9^{\circ}$ de una Institución Educativa (I.E) Pública de la ciudad de Tunja. El grupo está compuesto por 37 estudiantes, 20 niños y 17 niñas con edades entre los 14 a 17 años. La I. E Pública, recibe población de nivel socioeconómico de estratos 1, 2 y 3; los estudiantes viven en familias de distintos tipos: nucleares, extensa, mono parental paterna o materna, recontruidas. A partir de la observación insitu en las clases de inglés, se pudo percibir las características sociales de los estudiantes. El grupo de estudiantes es muy activo, por lo cual algunas veces recae en brotes de indisciplina, es decir, por ser un grupo grande, los estudiantes se entretienen con facididad conversando con sus compañeros, viendo sus celulares, etc., generando un ambiente de aprendizaje algo tenso; dicho de otra manera, 
la indisciplina del grupo, en ciertas ocasiones, obstaculiza el ambiente de aprendizaje. En cuanto al nivel de lengua inglesa, es un grupo heterogéneo, cada estudiante posee un nivel de lengua diferente, algunos estudiantes están interesados por el aprendizaje del inglés, otros no, dicho aspecto es reflejado en el desempeño académico de los discentes.

La muestra con la que se desarrolló la propuesta pedagógica, fueron 6 estudiantes de grado $9^{\circ}$, con edades entre 14 y 16 años de edad, 4 niños y 2 niñas que quisieron participar en el proceso pedagógico, ya que se desarrolló en horas extra escolares, teniendo en cuenta que dentro de la jornada escolar ordinaria, no se podía interrumpir a los estudiantes de sus actividades académicas de las otras áreas. Durante el desarrollo de las secuencias didácticas algunos estudiantes tenían sus dispositivos móviles o celulares que habitualmente llevaban a clase en el horario habitual. En cuanto al nivel de lengua, todos los estudiantes poseían un nivel diferente a pesar de ser del mismo grado. Algunas apreciaciones de los mismos participantes sobre el motivo a participar en las clases de inglés, fue el deseo de aprender más de este idioma, por lo tanto, los estudiantes demostraron motivación intrínseca por el desarrollo pedagógico. En cuanto a la parte social de los estudiantes, muestra de investigación, se puede decir que algunos de ellos son espontáneos y sociables al momento de hablar en inglés, y otros demuestran actitud de timidez o pena.

Los instrumentos usados en esta investigación suministraron información cualitativa y cuantitativa para responder a la pregunta: ¿Qué revela el uso de la aplicación grabadora de voz del celular, como estrategia didáctica en la producción oral en inglés, en estudiantes de grado $9^{\circ}$ de una Institución Educativa pública en la ciudad de Tunja- Boyacá?

Inicialmente, se recogió información con un pre-test (Tabla 1) para evaluar el nivel de producción oral en inglés en el que se encontraban los estudiantes muestra de la investigación; enseguida, se realizó la implementación de la estrategia didáctica, por medio de una secuencia didáctica dividida en seis talleres con su respectivo análisis del proceso de producción oral en inglés, hecho a través de una rúbrica de evaluación: contenido, vocabulario, uso gramatical, habilidad oral (pronunciación, entonación y fluidez), interacción y audibilidad (MEN, 2016) (Tabla 2). Al final de la implementación del proceso, se aplicó un cuestionario y una entrevista a los estudiantes para identificar aspectos relacionados con la producción oral, el aprendizaje cooperativo y uso del celular - aplicación Grabadora de Voz (Tabla 2) y, a su vez, se aplicó un post test (Tabla 1) para conocer el nivel de producción oral en inglés de los estudiantes al terminar la aplicación de la estrategia didáctica. Estos instrumentos permitieron encontrar información relevante sobre el desempeño de los estudiantes en su producción oral en inglés, a través del uso de la aplicación Grabadora de Voz.

Tabla 1

Pre-Post Test

\begin{tabular}{ll}
\hline Preguntas entrevista Pre Test & Preguntas entrevista Post Test \\
\hline What's your name? & Could you tell me something about yourself? \\
How old are you? & Taking into account the topic: Emotional \\
Where do you live? & Intelligent, could you describe your personal \\
What are your hobbies or favorites activities? & qualities? \\
What's your favorite singer or actor? Why? & What are your duties at your home or at school? \\
Could you tell me what activities did you do on & $\begin{array}{l}\text { Do you think you have been a good citizen? For } \\
\text { example, have you ever explained a difficult }\end{array}$ \\
your vacation? & maths problem or English topic to a classmate? \\
& $\begin{array}{l}\text { What was the most interesting topic that did you } \\
\text { study in the workshops? Why? }\end{array}$ \\
\hline
\end{tabular}

Fuente: Adaptado de Valencia, L. Y. (2015) 
Tabla 1

Rúbrica de evaluación de producción oral en inglés

\begin{tabular}{|c|c|c|c|c|}
\hline CRITERIA & $\begin{array}{l}\text { Low performance } \\
\text { (1.0 to 2.9) }\end{array}$ & $\begin{array}{c}\text { Basic performance } \\
\text { (3.0 to } 3.9 \text { ) }\end{array}$ & $\begin{array}{c}\text { High performance } \\
\text { (4.0 to } 4.5 \text { ) }\end{array}$ & $\begin{array}{c}\text { Superior } \\
\text { performance } \\
\text { (4.6 to } 5.0)\end{array}$ \\
\hline Content & $\begin{array}{l}\text { Has difficulty covering } \\
\text { the expected content } \\
\text { points. }\end{array}$ & $\begin{array}{l}\text { Covers with some } \\
\text { difficulty parts of the } \\
\text { intended topic, but } \\
\text { the message is not } \\
\text { badly affected. }\end{array}$ & $\begin{array}{l}\text { Covers the expected } \\
\text { content } \\
\text { successfully. }\end{array}$ & $\begin{array}{l}\text { Easily covers the } \\
\text { expected content } \\
\text { successfully. }\end{array}$ \\
\hline Vocabulary used & $\begin{array}{l}\text { Has difficulty using } \\
\text { vocabulary related to } \\
\text { the topic. }\end{array}$ & $\begin{array}{l}\text { Uses with a certain } \\
\text { amount of difficulty } \\
\text { some appropriate } \\
\text { vocabulary related to } \\
\text { the subject studied, } \\
\text { but the learner can } \\
\text { make himself/herself } \\
\text { understood. }\end{array}$ & $\begin{array}{l}\text { Uses vocabulary } \\
\text { which is } \\
\text { appropriate to the } \\
\text { subject studied. }\end{array}$ & $\begin{array}{l}\text { Easily uses extensive } \\
\text { and appropriate } \\
\text { vocabulary related to } \\
\text { the subject studied. }\end{array}$ \\
\hline $\begin{array}{l}\text { Use of English: } \\
\text { grammar }\end{array}$ & $\begin{array}{l}\text { Has difficulty using } \\
\text { grammar rules related } \\
\text { to the subject; has } \\
\text { problems expressing } \\
\text { himself/herself clearly. }\end{array}$ & $\begin{array}{l}\text { Uses with some } \\
\text { difficulty grammar } \\
\text { rules related to the } \\
\text { subject to make } \\
\text { himself/herself } \\
\text { understood. }\end{array}$ & $\begin{array}{l}\text { Uses correctly most } \\
\text { of the grammar } \\
\text { rules related to the } \\
\text { subject and } \\
\text { expresses } \\
\text { himself/herself } \\
\text { clearly. }\end{array}$ & $\begin{array}{l}\text { Uses grammar rules } \\
\text { related to the } \\
\text { subject extensively } \\
\text { and expresses } \\
\text { himself/ herself } \\
\text { clearly. }\end{array}$ \\
\hline $\begin{array}{l}\text { Oral ability } \\
\text { (Pronunciation/ } \\
\text { Intonation / Fluency) }\end{array}$ & $\begin{array}{l}\text { Speech is impossible to } \\
\text { understand or hard to } \\
\text { follow due to very poor } \\
\text { pronunciation, } \\
\text { intonation of key } \\
\text { words and slow } \\
\text { hesitant speech. }\end{array}$ & $\begin{array}{l}\text { Even though speech } \\
\text { is difficult to } \\
\text { understand, the } \\
\text { speaker has the } \\
\text { creativity to make } \\
\text { himself/herself } \\
\text { understood. }\end{array}$ & $\begin{array}{l}\text { Speech is generally } \\
\text { well pronounced } \\
\text { and fluid, with a few } \\
\text { hesitations. } \\
\text { However, the } \\
\text { speaker has some } \\
\text { pronunciation and } \\
\text { intonation } \\
\text { problems. }\end{array}$ & $\begin{array}{l}\text { Speech is well } \\
\text { articulated, } \\
\text { pronounced and } \\
\text { fluid, with very few } \\
\text { or no hesitations. It } \\
\text { is easy to understand } \\
\text { and uses correct } \\
\text { intonation. }\end{array}$ \\
\hline $\begin{array}{l}\text { Interaction and } \\
\text { audibility }\end{array}$ & $\begin{array}{l}\text { Has difficulty } \\
\text { maintaining } \\
\text { appropriate interaction } \\
\text { with audience or } \\
\text { speaking partner, } \\
\text { which impedes } \\
\text { communication. } \\
\text { Speaks very softly, } \\
\text { making it extremely } \\
\text { hard to hear and } \\
\text { impeding } \\
\text { understanding. }\end{array}$ & $\begin{array}{l}\text { Sometimes maintains } \\
\text { appropriate } \\
\text { interaction with } \\
\text { audience or speaking } \\
\text { partner, but } \\
\text { communication is } \\
\text { occasionally } \\
\text { impeded. Often } \\
\text { speaks very softly, } \\
\text { making it hard to } \\
\text { hear and therefore } \\
\text { frequently impeding } \\
\text { understanding. }\end{array}$ & $\begin{array}{l}\text { Interaction with } \\
\text { audience or } \\
\text { speaking partner is } \\
\text { appropriate most of } \\
\text { the time, although } \\
\text { some mistakes are } \\
\text { made. Level of } \\
\text { speech in terms of } \\
\text { sound is well } \\
\text { adjusted to setting } \\
\text { and surroundings } \\
\text { most of the time. }\end{array}$ & $\begin{array}{l}\text { Always maintains } \\
\text { appropriate } \\
\text { interaction with } \\
\text { audience or speaking } \\
\text { partner, which } \\
\text { facilitates } \\
\text { communication. } \\
\text { Level of speech in } \\
\text { terms of sound is } \\
\text { well adjusted to } \\
\text { setting and } \\
\text { surroundings, } \\
\text { facilitating } \\
\text { understanding. }\end{array}$ \\
\hline
\end{tabular}

Fuente: MEN (2016)

Los instrumentos de recolección de información: pre y post test, y las grabaciones de los estudiantes en las actividades orales durante el desarrollo de la secuencia didáctica, se evaluaron a partir de la rúbrica de Speaking que sugiere el MEN (2016) en los libros guía del docente. Como se mencionó anteriormente, la rúbrica de 
producción oral evalúa cinco aspectos: el contenido, el vocabulario, la gramática, la habilidad oral (pronunciación, entonación y fluidez), y la interacción y audibilidad. Al mismo tiempo, cada uno de estos criterios posee una clasificación de nivel: Low performance, es decir, desempeño bajo, se ubica en un rango de 1.0 a 2.9; Basic performance, en español, el desempeño básico que corresponde a un rango de 3.0 a 3.9; High performance, que significa desempeño alto y finalmente Superior performance, igual a desempeño superior. Los rangos numéricos de la rúbrica de evaluación de producción oral, pertenecen a los mismos criterios de evaluación establecidos en el ámbito escolar a la que pertenece la I.E.

La propuesta pedagógica se centró en el diseño, planeación y evaluación de una secuencia didáctica enfocada en una metodología de trabajo cooperativo, ya que como lo menciona Kagan (como se citó en D'Olivares, 2013) los grupos de aprendizaje cooperativo permiten a los estudiantes trabajar en equipo con otros compañeros que han adquirido mayor competencia comunicativa con el idioma, haciendo que haya un compartir de conocimientos.

Según Ortíz (2014) "la didáctica es la ciencia de aprender, por consiguiente, la didáctica propicia la actividad social, con autonomía del profesor, del estudiante y de la institución educativa para construir saberes y transformar la realidad" (p. 79), en palabras de Medina (como se citó en Ortíz, 2014) una didáctica aplicada en el aula abarca los procesos formativos y el conocimiento.

Por otra parte, Díaz (1998) define estrategia didáctica como los "procedimientos y recursos que utiliza el docente para promover aprendizajes significativos, facilitando intencionalmente un procesamiento del contenido nuevo de manera más profunda y consciente" (p. 19). A su vez, Tobón, S., Pimienta, J., y García, J. (2010) define estrategia didáctica como "el procedimiento que el agente de enseñanza utiliza en forma reflexiva y flexible para promover el logro de aprendizaje significativo en los estudiantes" (p. 7).

La secuencia didáctica, según Tobón et al (2010), es un conjunto articulado de actividades de aprendizaje y evaluación, con la mediación de un docente; buscan el logro de determinadas metas educativas, considerando una serie de recursos. La secuencia didáctica se diseñó y se aplicó desde un trabajo por competencias. Como lo argumenta Tobón et al (2010), se propone que los estudiantes desarrollen competencias para desenvolverse en la vida, por lo que es necesario la apropiación de contenidos en las diversas asignaturas, en el caso específico de este proceso investigativo, aunque el enfoque era la comunicación oral en inglés, se valió como excusa el trabajo interdisciplinario con áreas como ética y valores y ciencias sociales, con el fin de buscar procesos de formación y aprendizaje en torno a las competencias establecidas y relacionadas con el contexto de los estudiantes.

Los temas que se desarrollaron en los talleres contribuyeron a la reflexión de los estudiantes, ya que favorecieron procesos de convivencia pacífica, promovieron valores éticos y su capacidad de conciliación. Los títulos de los talleres desarrollados fueron: 1) Preséntate y presenta a otros, 2) La inteligencia emocional: descubriendo cualidades positivas y negativas de la personalidad, 3) Derechos y deberes de los niños, 4) ¿Has sido un modelo de ciudadano?, 5) Vamos a parar el abuso verbal! y, por último, 6) Resuelve sabiamente los problemas personales. Estos temas fueron adaptados del libro Way to go! que propone el MEN (2016) para la enseñanza aprendizaje del inglés en instituciones públicas.

Para el desarrollo de la secuencia didáctica se empleó el taller como sistema de enseñanza - aprendizaje propuesto por Ander - Egg (1999), quien argumenta que por medio del taller se aprende haciendo, permite una metodología participativa (docente- estudiantes), donde se aprende a desarrollar conductas, actitudes y comportamientos participativos; el taller es un entrenamiento de trabajo interdisciplinario que implica y exige el desarrollo de las actividades por medio de un trabajo grupal; permite integrar tres aspectos importantes la docencia, la investigación y la práctica. 
Enseguida se diseñaron seis talleres con su respectivo formato de planeación (anexo 1) y la guía del estudiante (anexo 2), cada formato de planeación de la secuencia didáctica tenía aspectos como: objetivo a lograr en el taller, los estándares que se deseaban desarrollar en cada taller, ya fuera lectura, escritura, escucha, monólogos, conversación; el DBA (Derechos Básicos de Aprendizaje) (MEN, 2016) que se esperaba alcanzar y las competencias a desarrollar expuestas en indicadores del saber, el hacer y el ser, y el área con la que se aplicaba la transversalidad.

En cuanto al seguimiento, planeación, implementación y evaluación del proceso didáctico de las clases, se desarrollaron los siguientes pasos: 1) exploración: que consiste en las actividades de preparación para el aprendizaje, establecer el objetivo, reconocimiento de conocimientos previos, este primer momento se realizó por medio de tongue twisters, posters de inferencia, videos, cuestionarios, lectura de imágenes, preguntas de exploración, etc.; 2) estructuración: consiste en la contextualización y modelación del nuevo conocimiento, es decir, la enseñanza explícita del docente, para este paso, la docente contextualizó a los estudiates las nuevas estructuras gramaticales por medio de lecturas, juegos de mesa, nube de palabras, unión de preguntas y respuestas, completar oraciones, etc.; 3) práctica: radica en la práctica guiada de la ejercitación y la práctica independiente, las actividades de práctica fueron ejercicios de comprensión de lectura, cuestionarios, ejercicios de escucha, mesas redonda, ejercicios de escritura, etc.; 4) transferencia: es la aplicación del aprendizaje, luego de desarrollar diversas actividades de lectura, escritura, escucha, finalmente se ejercitó la habilidad de habla en inglés, que en este caso, fue el nucleo central de la investigación, donde se usó la grabadora de voz del celular, conviertiendose en una herramienta que propició la producción oral en inglés en los estudiantes, por medio de actividades cooperativas como juego de roles, exposiciones, presentaciones de posters, programas de radio, las cuales fueron planeadas por los estudiates, luego grabadas en sus dispositivos móviles y por último socializadas a sus compañeros como se muestra en el anexo 1 y anexo2; por último, 5) seguimiento: reflexión en qué medida se cumplieron los objetivos propuestos en el taller, no solo con el desarrollo de las habilidades comunicativas orales, sino los correspondientes al tema tratado en cada taller, además se emplea la autoevaluación, coevaluación y hetero evaluación.

\section{Resultados}

Para el análisis de la información en esta investigación se tomó como base la teoría fundamentada (Grounded Theory). En palabras de Strauss y Corbin (como se citó en Ortíz, 2015) la teoría fundamentada "es una manera de pensar la realidad social y de estudiarla" (p. 116). Hernández et al. (2014) argumentan que el propósito de la teoría fundamentada es desarrollar teoría basada en datos empíricos, es decir, sostiene que las teorías son sustantivas o de rango medio que se construyen en el propio campo de acción y ayudan a explicar el mundo real de la educación (Ortíz, 2015). Por consiguiente, se realizaron las siguientes etapas: 1) recolección de los datos por medio de observaciones de práctica docente, encuesta diagnóstica, aplicación de pre y post test de nivel de producción oral, audios grabados en el celular de los estudiantes durante los 6 talleres y cuestionario a estudiantes; 2) organización de los datos de información: establecer criterios de organización; 3) preparación de los datos para el análisis: transcribir datos verbales en texto escrito, recogidos en las grabaciones de los estudiantes, apuntes del diario de campo, transcribir entrevistas del pre y post test, evaluar los audios por medio de la rúbrica de producción oral en inglés; sintetizar datos recogidos en el cuestionario de los estudiantes, transcribir la entrevista final de los estudiantes 4) revisión y clasificación de los datos obtenidos con cada instrumento; 5) elección de las unidades o categorías de análisis a la luz de la revisión los datos; 6) descripción de conceptualizaciones, definiciones, significados o ejemplos, y a su vez, agrupar y relacionar categorías y finalmente, generar teorías o explicaciones. En esta investigación, se generaron explicaciones con base en la información recolectada, que apuntó a dar respuesta a la pregunta de investigación. 
A través del cuestionario a los estudiantes, se quiso conocer sus percepciones frente a la implementación de la secuencia didáctica mediada por las TIC, y su influencia en la producción oral en inglés. El cuestionario estuvo dividido en tres aspectos de análisis: el uso de la aplicación Grabadora de Voz del celular; la producción oral en inglés y el aprendizaje cooperativo.

La primera pregunta que se hizo fue sobre si el uso de la aplicación Grabadora de voz del celular, permitió el fortalecimiento de su producción oral en inglés. De acuerdo con las respuestas, el $100 \%$ de los estudiantes respondieron que sí y algunos de sus argumentos fueron:

- "Podiamos escuchar nuestros errores y corregirlos" (Cuestionario - E1)

- "porque me ayudó a analizar cuál de las palabras pronuncié mal y cuáles fueron mis errores" (Cuestionario $-E 2)$

En segundo lugar, se preguntó a los estudiantes si recomendarían el uso del celular en ambientes escolares para la práctica de producción oral en inglés. De la muestra, 5 estudiantes respondieron sí y solo 1 estudiante respondió que no, algunos de los argumentos de quienes contestaron afirmativamente fueron:

- "es buena herramienta para aumentar el ingles en los estudiantes" (Cuestionario - E1)

- "porque puede aportar a la fluidez y reconocimiento de errores propios" (Cuestionario - E5)

- Por el contrario, E3, quien no recomendaría el uso del celular dijo:

- "seria distinto a un grupo pequeño de compañeros, y un grupo grande seria incomodo" (Cuestionario E3)

- La estudiante que argumenta lo negativo en el uso del celular en ambientes escolares, argumenta en la entrevista:

- "pues es que con un grupo grande lo que ocasiona es indisciplina, en cambio un grupo pequeño es más que todo el apoyo del uno al otro y como que más fluido, porque hay menos personas, si...con un grupo grande por lo menos yo, no me voy a entender porque hay mucha indisciplina, unos en su cuento, otros en otro y entonces como que no hay conexión para el trabajo" (Entrevista - E3)

En tercer lugar, se preguntó a los estudiantes si se veían motivados a utilizar el celular como herramienta pedagógica, para mejorar su nivel de producción oral en inglés, en contextos extracurriculares, ya fuera en sus hogares, en el centro comercial, en paseos, etc. De los estudiantes muestra de la investigación, 1 estudiante marcó la opción Siempre, 4 estudiantes escogieron la opción Usualmente, y 1 estudiante seleccionó la opción Rara vez. De acuerdo con la entrevista, los estudiantes argumentaron:

- (Siempre) "pues como para mejorar el léxico, también el traductor es como muy necesario pues ahí la opción auditiva y pues el traductor..." (Entrevista - E6)

- (Usualmente) "pues porque yo siempre hee...pues primero, pues a veces pongo música, o utilizo Duolingo pues para ir mejorando el inglés, vocabulario" (Entrevista - E4)

- (Rara vez) "Porque es que cuando uno va a paseo, más que todo se dedica a otras cosas y entonces como que no se puede concentrar para hacer el trabajo y en la casa como que se distrae en otras actividades, en cambio en el colegio estamos haciendo algo específico y nos podemos concentrar" (Entrevista - E3)

Por último, respecto al uso del celular en ambientes escolares, se les preguntó a los estudiantes, si les había gustado interactuar con la aplicación Grabadora de Voz del celular, como estrategia para mejorar su producción oral en inglés. El $100 \%$ de los estudiantes respondieron que sí, algunas respuestas presentadas fueron las siguientes:

- "fue un nuevo método y además muy bueno" (Cuestionario - E1)

- "por que al escucharme pude identificar mis errores" (Cuestionario - E2) 
- "porque nos ayuda a escuchar errores que nosotros mismos no identificamos" (Cuestionario - E5)

En relación con la producción oral en inglés, al preguntarles a los estudiantes cómo se sienten a la hora de hablar en inglés, dos estudiantes escogieron la opción Muy cómoda, uno de ellos registró:

- "porque me siento como con más fluides, pronunciando y escuchando" (Cuestionario - E2)

- Los otros cuatro estudiantes seleccionaron la opción Cómodo. En síntesis, ellos argumentan que se sienten cómodos porque han mejorado su fluidez en el idioma, tiene buen vocabulario, sienten acompañamiento de la docente y de sus compañeros, como lo sustentan:

- "porque en las últimas clases e mejorado mi fluides en ingles" (Cuestionario - E1)

- "porque tengo buen vocabulario para tener fluidez a la hora de hablar" (Cuestionario - E6)

Con base en los criterios de la rúbrica de producción oral, se les preguntó a los estudiantes, si consideraban que su pronunciación en inglés había mejorado; el 100\% de los estudiantes respondieron que sí, algunas respuestas son las siguientes:

- "antes no savia pronunciar muy bien las palabras ahora me fluyen mas al pronunciarlas gracias a todas las actividades y grabaciones" (Cuestionario - E3)

- "por que al pronunciarlo al principio tuve errores pero con la ayuda de la profesora mis compañeros y la tecnología, he podido pronunciar mejor" (Cuestionario - E2)

En el mismo aspecto de producción oral se encuentra lo pertinente a la fluidez, con el objetivo de conocer si los estudiantes consideraban que en este momento hablan con mayor fluidez que antes de comenzar los talleres, nuevamente el $100 \%$ de los estudiantes respondieron que sí, algunos argumentos presentados son los siguientes:

- "ya se como pronunciar las palabras mejor y se como construir textos oralmente" (Cuestionario - E3)

- "porque en algunas palabras no sabia la pronunciación y lo decía como siendo lo mas lógico y cuando me daba cuenta estaba erróneo asi que fui mejorando tambien la fluidez" (Cuestionario - E5)

En relación con la gramática y el vocabulario, se les preguntó ¿Con qué frecuencia usa las estructuras gramaticales y vocabulario estudiado en clase, a la hora de hablar en inglés?, el $100 \%$ de los estudiantes respondieron Usualmente. Dicho resultado se vio reflejado en la actividad del post test, en el cual, la mayoría de los estudiantes utilizaron parte del vocabulario y las estructuras gramaticales acorde con el tema tratado. Igualmente, en entrevista con los estudiantes argumentaron:

- "pues porque cuando estoy con mis primos o mis amigos, a veces nos gusta hablar en inglés para retarnos a hablar en otro idioma, y en mi casa, mi mamá me...a veces me hace preguntas en inglés como por mejorar y por eso lo utilizo usualmente (Entrevista -E1)

En otra de las preguntas se quiso conocer si ellos comprendían clara y espontáneamente la intervención de su interlocutor cuando le habla en inglés. Al respecto, dos estudiantes, respondieron Siempre; 3 estudiantes, respondieron Usualmente y un estudiante, respondió Algunas veces. De acuerdo con la entrevista, los estudiantes sustentaron:

- (Siempre) "pues todo, todo, no es que le entiende no profe, pero si algunas veces, o sea, casi siempre alcanzo a entender qué es lo que hay que hacer en las actividades asi" (Entrevista - E4)

- (Usualmente) "pues yo cuando hablan en inglés yo intento... yo intento... no se... escuchar algunas palabras conocidas, buscar el significado e intentar interpretar lo que quiere decir la persona" (Entrevista - E5) 
- (Algunas veces) “Porque es que a veces como que uno está de pronto distraído o de pronto sumercé habla con fluidez y uno se queda en una palabra como qué entendí y se pierde el resto entonces es...en cambio hay cosas que uno ya más o menos las va captando desde que lo dice" (Entrevista - E3)

Teniendo en cuenta que el objetivo de los talleres no era solo el que los estudiantes pudieran hablar de los temas tratados, con la información allí suministrada, sino que ellos contribuyeran, según sus experiencias o conocimiento, en el cuestionario se incluyó la siguiente pregunta: ¿Cuando usted habla en la clase de inglés, lo hace sobre el tema indicado y aporta nuevas ideas? Todos los estudiantes escogieron la opción Usualmente. Este aspecto se reflejó en el desarrollo de varias actividades orales en los distintos talleres, ya que algunos estudiantes sólo se limitaron a desarrollar la actividad propuesta y no aportaron nuevas ideas acerca del tema. Igualmente, en la entrevista algunos señalaron:

- "Porque la mayoría de veces yo trato como de utilizar lo que es, y me gusta aportar porque no sé, hay temas en que uno le llama la atención y se acuerda y dice no a mí me dijeron esto, entonces me gusta ---, pero a veces se me olvida" (Entrevista -E3)

El último aspecto de análisis en la aplicación del cuestionario a los estudiantes, fue el relacionado con la metodología implementada en el desarrollo de la secuencia didáctica, es decir, el aprendizaje cooperativo.

Al preguntarles a los estudiantes si el trabajo cooperativo con otros compañeros, contribuyó en el fortalecimiento de su producción oral en inglés, el $100 \%$ de los estudiantes respondieron que sí. Algunos de sus argumentos fueron los siguientes:

- "porque el trabajo cooperativo con mis compañeros ha fortalecido mi trabajo y mi producción oral ya que me colaboran al uso de la vocalización de palabras al igual que yo a él” (Cuestionario - E2)

- "porque cuando se habla ingles en el grupo, damos opiniones, criticas constructivas, y correcciones de pronunciación" (Cuestionario - E5)

- "porque algunos compañeros tienen mejor aprendizaje de ingles y nos pueden ayudar" (Cuestionario E6)

Por otra parte, al preguntarles a los estudiantes si habían sentido un ambiente de aprendizaje agradable trabajando entre compañeros, se obtuvo como resultado, 4 estudiantes marcaron Siempre, 1 estudiante escogió Usualmente y 1 estudiante seleccionó Algunas veces. Algunas razones de los estudiantes que marcaron Siempre fueron:

- "porque cada uno aporta lo que sabe y opina sobre el tema y apoya" (Cuestionario - E5)

- "nos reíamos siempre de alguna cosa divertida que pasara y al aprender ingles nos entendíamos" (Cuestionario - E1)

- "al trabajar en grupo me ayuda a socializar, a aprender, a enseñar a mi compañero (a) al igual que ella a mi" (Cuestionario -E2)

El estudiante que escogió Usualmente, argumentó:

- "si porque hay ayuda entre los dos" (Cuestionario E4)

El estudiante que marcó Algunas veces, sustentó:

- "a veces habían compañeros que no manejaban el trabajo cooperativo, era muy difícil pero tambien habían ambientes colaboradores" (Cuestionario - E3)

Por otro lado, analizando la convivencia de los estudiantes, se les preguntó si habían podido resolver los conflictos presentes en el trabajo cooperativo, obteniendo como resultado, 5 estudiantes escogieron la opción 
sí y 1 estudiante seleccionó la opción no. Algunas opiniones dadas por los estudiantes que escogieron la opción positiva fueron:

- "La verdad nunca tuve un problema mayor con mis compañeros" (Cuestionario - E1)

- "porque cuando una persona esta cegada por la rabia solo se deja respirar, luego si se habla y se ayuda incluyendo temas de los talleres" (Cuestionario - E5)

- "nos ponemos de acuerdo en las decisiones" (Cuestionario -E4)

- En cuanto al estudiante que escogió la opción no, su argumento fue:

- "la gente no va a cambiar su manera de trabajar y aunque es difícil hago mi parte y esto destruye la cooperación? (Cuestionario - E3)

Con relación a este aspecto, se les preguntó a los estudiantes qué tipo de conflictos tuvieron en el trabajo cooperativo y cómo los resolvieron, de acuerdo con la entrevista, los estudiantes respondieron:

- "Pues que a veces no les gustaba que digamos uno los corrigiera, y pues uno notaba ahí el cambio de actitud, pero después escuchándonos en el celular, ahí ya decían que sí, que sí tenía razón y pues ya salía mejor el trabajo" (Entrevista-E4)

- "pues cuando hicimos los grupos, pues cada uno tenía una opinión, si y entonces, uno tenía como de juntarlas y escuchar la opinión de los otros, para poder hacer bien el trabajo...por ejemplo una persona tenía más conocimiento que los otros, entonces si uno tenía pues el conocimiento, entonces le decía, pues si tú sabes o algo, uno le pedía la opinión y uno lo aceptaba, pero nunca hubo conflictos" (Entrevista - E6)

Continuando con los aspectos que involucran el aprendizaje cooperativo, se les preguntó a los estudiantes si era posible identificar una actitud de liderazgo y confianza entre los integrantes el trabajo cooperativo, el 100\% de los estudiantes respondieron que sí, algunos de sus argumentos fueron:

- "hay veces la persona que le fluye mas el ingles es colaboradora con los demas para tener un mejor resultado" (Cuestionario -E3)

Por último, se les preguntó a los estudiantes si hubo compromiso y responsabilidad individual para hacer su tarea dentro del grupo cooperativo, el $100 \%$ de los estudiantes respondieron de forma afirmativa. Algunas opiniones de los estudiantes fueron:

- "por que se toma en serio el trabajo además de trabajar en equipo" (Cuestionario - E2)

Finalmente, como valor agregado a este cuestionario, se les preguntó a los estudiantes si los temas trabajados en los talleres contribuyeron en su formación personal, dando como resultado que el $100 \%$ de los estudiantes afirmaron que sí. Algunos argumentos fueron:

- "ya que he aprendido a manipular mejor este idioma a conocer palabras nuevas a socializar con mis compañeros y a realizar mejor mis trabajos de ingles" (Cuestionario - E2)

\section{Conclusiones}

La grabadora de voz, más que una aplicación para guardar información, es una herramienta que permitió a los estudiantes autoevaluarse y reducir su ansiedad en el momento de desarrollar actividades orales. En cuanto a esta investigación refiere, el uso de la grabadora de voz del celular, como mediación TIC para el desarrollo de la producción oral en inglés, cumplió con sus funciones básicas como lo es capturar sonido y almacenarlo en la memoria del celular o en una sim card y luego poder reproducir dicho audio. Pareciera un proceso simple, pero detrás de este sistema de uso, se obtuvo hallazgos importantes, ya que los estudiantes generaron reflexiones de su propio proceso de aprendizaje, en palabras de Schmitt (2010) "las estrategias metacognitivas son aquellos 
procesos que los alumnos utilizan conscientemente para supervisar o gestionar su aprendizaje de una lengua" (p. 166). El hecho que los estudiantes pudieran escucharse a sí mismos, no sólo produjo el reconocimiento de su propia voz, sino que pudieron identificar sus aciertos y desaciertos en su producción oral en inglés, lo cual contribuyó en su proceso de autoevaluación. Como lo sustenta Sluijsmans, Dochy, \& Moerkerke (MEN, 2016) la autoevaluación es hecha cuando el estudiante reflexiona acerca de su propio aprendizaje, si el estudiante es capaz de hacer juicios a sus hábitos y estrategias de aprendizaje, vocabulario, entre otros aspectos, se logra que se involucre activamente en su educación.

De igual manera, la secuencia didáctica mediada por el uso de la grabadora de voz del celular, influyó en la reducción de los grados de ansiedad de los estudiantes, al momento de hablar en inglés, ya que como lo sustenta Rubio (como se citó en Cordero \& Morales, 2016) al crear un ambiente de aula positivo, donde se ayuda a eliminar sentimientos de inseguridad y crear un ambiente de cooperación, y no de competición entre los estudiantes, además de desarrollar estrategias de corrección de errores que hagan encontrar oportunidades de mejoramiento, estas acciones posibilitan reducir la ansiedad a la hora de hablar en inglés.

La grabadora de voz del celular es una aplicación sencilla, pero puede conducir a generar aprendizaje cooperativo. Durante este estudio se encuentra un proceso que inicia con actividades individuales que, posteriormente, promueve el aprendizaje cooperativo a través de actividades macro de producción oral, planteadas dentro de los talleres, como fue hacer exposiciones y diálogos, etc., en las que los estudiantes se congregaron para planear, desarrollar y evaluar dichos ejercicios, mediante el uso de la grabadora de voz.

Vale la pena recordar que el aprendizaje de una lengua, como lo establece Cassany, D., Luna, M., y Sanz, G, (2003) es la adquisición y el desarrollo de las estrategias necesarias para comunicarse, una de ellas, es generar interacción comunicativa entre los sujetos, según lo sustenta Kagan (como se citó en D'Olivares, 2013) los grupos de aprendizaje cooperativo permite a los estudiantes trabajar en equipo con otros compañeros que han adquirido mayor competencia comunicativa con el idioma, haciendo que haya un compartir de conocimientos, como lo sustentaron los estudiantes en el cuestionario

El celular más allá de entretener puede fortalece la producción oral en inglés. Aunque algunos docentes y padres de familia consideran frecuentemente el celular como un dispositivo móvil que distrae los procesos pedagógicos, al igual que los resultados de algunas investigaciones, como la realizada por Chen y Yan (como se citó en Rodríguez \& Juárez, 2017) quien encontró algunos componentes que pueden afectar el aprendizaje, cuando se tiene un dispositivo móvil en el aula, también se encuentran experiencias investigativas que muestran una visión positiva en cuanto al uso pedagógico de los dispositivos móviles, como en el caso de Al Hamdani (como se citó en Rodríguez \& Juárez, 2017) encontró que los dispositivos ayudaron a los participantes a promover habilidades de pensamiento y a cooperar entre pares, de igual forma, Fried (como se citó en Rodríguez \& Juárez, 2017) menciona que los dispositivos móviles pueden incrementar la motivación de los estudiantes dentro del aula de clase.

En el caso concreto de esta investigación, sin necesidad de internet, se logró movilizar conocimiento por medio de la aplicación de la grabadora de voz del celular, con la que los estudiantes ejercitaron su producción oral en inglés, teniendo en cuenta temas contextualizados según plan de área diseñado para el grado $9^{\circ}$.

\section{Referencias bibliográficas}

Ander-Egg, E. (1999). El taller: Una alternativa de renovación pedagógica. Argentina: Editorial Magisterio del Río de la Plata.

Camacho, B. (2003). Metodología de la Investigación Científica: Un camino fácil de recorrido para todos. Tunja: Editorial Universidad Pedagógica y Tecnológica de Colombia. 
Cassany, D., Luna, M., y Sanz, G. (2003). Enseñar lengua. España: Editorial GRAÓ, de IRIF, S.L.

Díaz, F. (1998). Una aportación a la didáctica de la historia. La enseñanza aprendizaje de habilidades cognitivas en el bachillerato. Perfiles educativos, 20 (82), 40-66.

D'olivares, N. (2013). Cooperative Learning: Another Way to Learn Together. Enletawa Journal, (6), 59-72. Recuperado de http://revistas.uptc.edu.co/index.php/enletawa_journal/article/view/3082

Elliott, J. (1990). La investigación - acción en educación. Morata. Recuperado de: http://www.terras.edu.ar/biblioteca/37/37ELLIOT-Jhon-Cap-1-y-5.pdf

Gutiérrez, P., Fernández, A., y Tabasso, E. (2017). Humanizar la utilización de las TIC en educación. Revista de Investigación en Educación, 15 (1), 80-82. Recuperado de http://reined.webs.uvigo.es/index.php/reined/article/view/205

Hernández, R., Fernández, C., y Baptista, M. (2014). Metodología de la investigación . México: Mc Graw Hill Education.

MEN. (2016). Derechos Básicos de Aprendizaje de Inglés. Bogotá D.C: Imprenta Nacional.

MEN. (2016). Orientaciones y principios pedagógicos: currículo sugerido de inglés. Bogotá D.C: Imprenta Nacional.

MEN. (2016). Way to go! 8th Grade Teacher's Guide. Bogotá D. C: Imprenta Nacional.

Ortíz, A. (2014). Currículo y didáctica. Bogotá: Ediciones de la U.

Ortíz, A. (2015). Desarrollo del pensamiento y las competencias básicas cognitivas y comunicativas - ¿Cómo formular estándares, logros e indicadores de desempeño? Bogotá: Distribooks Editores.

Robayo, J. O. (2016). Reducing Anxiety and Shyness for Oral Interaction Improvement in a Cooperative Learning Environment. Enletawa Journal, 9 (1), 53-65. Recuperado de: https://revistas.uptc.edu.co/index.php/enletawa_journal/article/view/7539

Rodríguez, J., y Juárez, J. P. (2017). Impacto del m-learning en el proceso de aprendizaje: habilidades y conocimiento. RIDE Revista Iberoamericana para la Investigación y el Desarrollo Educativo, 8 (15), 363386. doi: https://doi.org/10.23913/ride.v8i15.303

Schmitt, N. (Ed.). (2010). An Introduction to Applied Linguistics. London, Great Britain: Hodder Education.

Tobón, S., Pimienta, J., y García, J. (2010). Secuencias Didácticas: aprendizaje y evaluación de competencias. México: Pearson Educación.

Valencia, L. Y. (2015). La competencia oral en una L2 apoyada por el uso de TIC en educación media (Tesis de maestría). Recuperado de: http://ayura.udea.edu.co:8080/jspui/handle/123456789/1949 


\section{Anexo 1. Planeación Secuencia Didáctica - Taller 2}

Nombre del proyecto: El uso del teléfono celular como herramienta para incentivar la producción oral en inglés, en una Institución Educativa Pública

PLAN DE AULA ÁREA INGLÉS ${ }^{1}$

\begin{tabular}{|c|c|c|c|c|c|c|c|}
\hline Grado: $9^{\circ}-01$ & \multicolumn{2}{|c|}{$\begin{array}{l}\text { Fecha: } 13 \text { de mayo } \\
\text { de } 2019\end{array}$} & \multicolumn{3}{|c|}{ Área: humanidades- inglés } & Escenario: salón de clases & \multirow[b]{2}{*}{ Tiempo: 5 horas } \\
\hline \multicolumn{2}{|c|}{ 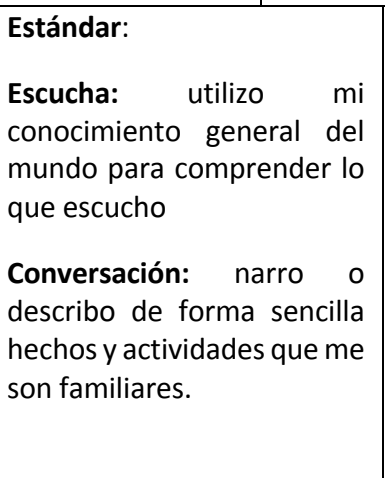 } & \multicolumn{3}{|c|}{$\begin{array}{l}\text { - DBA: intercambia } \\
\text { información acerca de } \\
\text { temas de interés } \\
\text { académico o general en } \\
\text { una conversación. }\end{array}$} & \multicolumn{2}{|c|}{$\begin{array}{l}\text { Competencias } \\
\text { Saber: reconoce el papel del lenguaje } \\
\text { positivo y negativo en la construcción de paz } \\
\text { en la comunidad } \\
\text { Hacer: presenta opiniones en el uso del } \\
\text { lenguaje positivo o negativo siguiendo un } \\
\text { plan de presentación preparado con } \\
\text { anterioridad } \\
\text { Ser: muestra respeto por la opinión } \\
\text { expresada por sus compañeros }\end{array}$} & \\
\hline 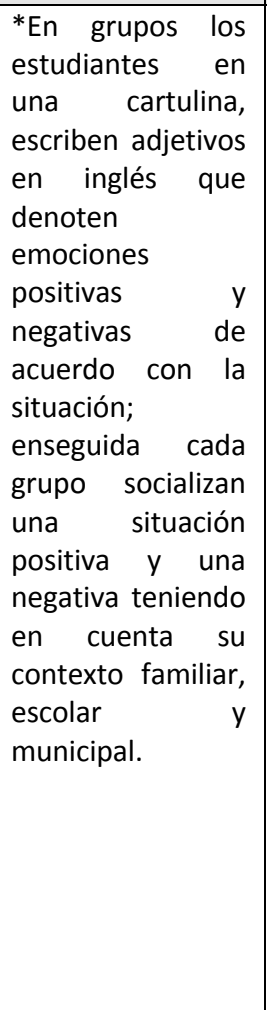 & \multicolumn{3}{|c|}{$\begin{array}{l}\text { * Los estudiantes identifican } \\
\text { el nuevo vocabulario por } \\
\text { medio de una nube de } \\
\text { palabras y clasifica las } \\
\text { palabras en cualidades } \\
\text { positivas y negativas. } \\
\text { *Cada estudiante selecciona } \\
\text { palabras del vocabulario } \\
\text { anterior para describir su } \\
\text { personalidad. } \\
\text { *En grupos los estudiantes } \\
\text { discuten sobre la definición } \\
\text { de inteligencia emocional y } \\
\text { socializan sus ideas. } \\
\text { *La docente sintetiza las } \\
\text { respuestas de los } \\
\text { estudiantes en una única } \\
\text { definición. } \\
\text { *En grupos los estudiantes } \\
\text { responden las siguientes } \\
\text { preguntas: -¿En Colombia } \\
\text { las personas tienen } \\
\text { inteligencia emocional? } \\
\text {-¿Ustedes creen que tienen } \\
\text { inteligencia emocional? }\end{array}$} & \multicolumn{2}{|c|}{$\begin{array}{l}\text { *En grupos de } 3 \\
\text { estudiantes, organizan } \\
\text { una breve presentación } \\
\text { cumpliendor los } \\
\text { siguientes parámetros: } \\
\text { concepto de inteligencia } \\
\text { emocional, por qué es } \\
\text { importante la } \\
\text { inteligencia emocional, } \\
\text { enseguida a partir de } 4 \\
\text { situaciones problema, } \\
\text { cada grupo escoge dos } \\
\text { situaciones y establece } \\
\text { los respectivos consejos } \\
\text { para solucionar dicha } \\
\text { situación. Finalmente } \\
\text { socializan la } \\
\text { presentación frente a la } \\
\text { docente y compañeros } \\
\text { de clase. }\end{array}$} & $\begin{array}{l}\text { *Hetero-evaluación } \\
\text { docente mediante la } \\
\text { observación en las diferentes } \\
\text { sesiones de clase, y por medio } \\
\text { de la rúbrica de producción } \\
\text { oral (pronunciación. fluidez y } \\
\text { uso del idioma), establecerá el } \\
\text { seguimiento de cada } \\
\text { estudiante; al igual que } \\
\text { analizará aspectos }\end{array}$ & $\begin{array}{l}\text {-Posters } \\
\text {-Fotocopias } \\
\text {-Celular } \\
\text {-Libro guía del } \\
\text { estudiante. } \\
\text {-Malla } \\
\text { autoevaluación. } \\
\text {-Rúbrica de evaluación } \\
\text { de producción oral en } \\
\text { inglés. }\end{array}$ \\
\hline
\end{tabular}

\footnotetext{
${ }^{1}$ Formato para la planeación de aula diseñada por el MEN “Programa Todos a Aprender” (MEN, 2006) y modificado para la Institución Educativa A.J.S.G.
} 


\begin{tabular}{|c|c|c|}
\hline & $\begin{array}{l}\text {-¿¿a inteligencia emocional } \\
\text { ayuda a ser un buen } \\
\text { ciudadano? } \\
\text { *Explicación de contenido } \\
\text { instruccional: modal verb: } \\
\text { should / shouldn't } \\
\text { *Identificación } \\
\text { situaciones en contexto: en } \\
\text { grupos, los estudiantes leen } \\
\text { tres situaciones problema e } \\
\text { identifican el correcto } \\
\text { comentario. } \\
\text { *Los estudiantes leen la } \\
\text { cuarta situación problema } \\
\text { para que ellos escriban el } \\
\text { consejo para solucionar el } \\
\text { problema. } \\
\text { *Los estudiantes escuchan } \\
\text { un audio sobre cómo los } \\
\text { ciudadanos colombianos } \\
\text { aplican la inteligencia } \\
\text { emocional y clasifican los } \\
\text { ítems en verdadero o falso } \\
\text { según corresponda. }\end{array}$ & $\begin{array}{l}\text { relacionados con } \text { el } \\
\text { aprendizaje cooperativo y el } \\
\text { uso de la aplicación de } \\
\text { grabadora de voz. }\end{array}$ \\
\hline
\end{tabular}

\section{Anexo 2. Taller 2}

\section{INSTITUCIÓN EDUCATIVA ANTONIO JOSÉ SANDOVAL GÓMEZ ENGLISH WORKSHOP \# 2}

NAME: Grade: $9^{\circ}-01$ Date:

I. VOCABULARY: look at the word cloud and find 9 positive qualities and 8 negative qualities

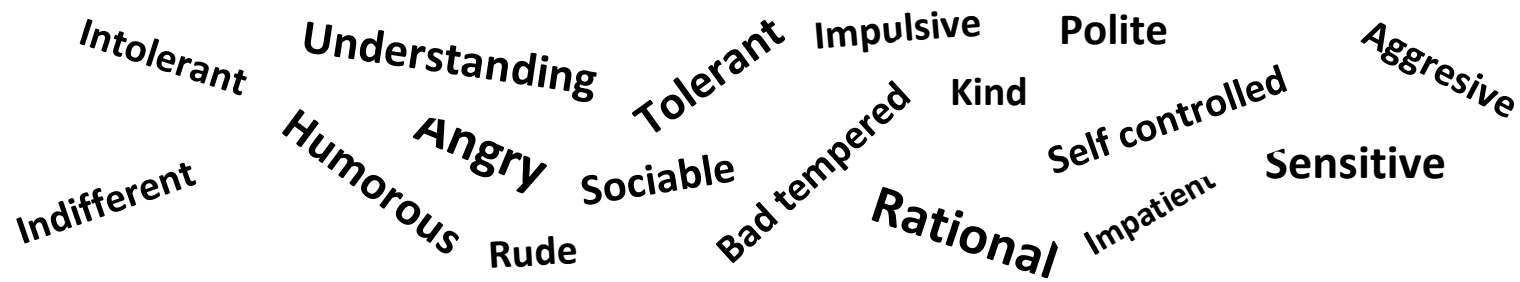

II. WRITING: select the personal qualities (positives or negatives) that describe you. Be honest!

III. SPEAKING: discuss with your partners: What is the definition of emotional intelligence?

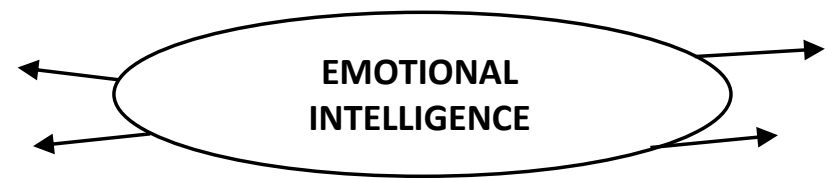

IV. SPEAKING: according to the definition of emotional intelligence, discuss the questions with your partners:

a. Do Colombian people have emotional intelligence?

b. Do you think you have emotional intelligence?

c. Does emotional intelligence help you to be a good citizen? 
V. Review the topic with your teacher:

\begin{tabular}{|c|l|}
\hline \multicolumn{2}{|c|}{ MODAL VERBS: SHOULD / SHOULDN'T } \\
\hline FUNTION & \multicolumn{1}{c|}{ EXAMPLE } \\
\hline \multirow{3}{*}{$\begin{array}{c}\text { To make suggestions or give } \\
\text { advice }\end{array}$} & *You should think positively \\
& *They should speak to the team coach \\
& *She should try to understand \\
& *He shouldn't be annoyed \\
\hline
\end{tabular}

VI. READING: some teenagers are sharing their experience online. Match the comments according to the situation.

\begin{tabular}{|c|c|c|}
\hline \multicolumn{3}{|c|}{$\begin{array}{l}\text { Forum: when People Hurt Your Feelings } \\
\text { This topic contains } 4 \text { replies, and was updated by Susi0072 one minute ago. }\end{array}$} \\
\hline TommiCoolguy & $\begin{array}{l}\text { Comment } 1 \text { Like. Replay Feb 23, } 2016 \text { 12:49 p.m } \\
\text { My best friend is angry with me. He said that I was } \\
\text { selfish because I didn't let him copy my homework. Now } \\
\text { he doesn't want to speak to me any more. }\end{array}$ & $\begin{array}{l}\text { a. Reply to comment } \\
\text { You should speak to the team coach } \\
\text { about their comments. You probably } \\
\text { just need more practice. }\end{array}$ \\
\hline I_love_football & $\begin{array}{l}\text { Comment } 2 \text { Like. Replay Feb 24, } 2016 \text { 5:10 p.m } \\
\text { I feel really bad today. The other players in my football } \\
\text { team say that I'm lazy and fat. They say I should run } \\
\text { faster. They make me feel I'm a bad player. }\end{array}$ & $\begin{array}{l}\text { b. Reply to comment } \\
\text { You should speak to your mum and } \\
\text { say you are sorry. You were rude to } \\
\text { her. She is just worried about you. You } \\
\text { should try to understand her. }\end{array}$ \\
\hline Karen2017 & $\begin{array}{l}\text { Comment } 3 \text { Like. Replay Feb 27, } 2016 \text { 6:00 p.m } \\
\text { I feel bad because I argued with my mom. She said that } \\
\text { I can't go to a party with my friends. So I said, ilt's not } \\
\text { fair! You're running my life! She started to cry. Should I } \\
\text { say sorry or just forget about it? }\end{array}$ & $\begin{array}{l}\text { c. Reply to comment } \\
\text { You should speak to him about the } \\
\text { importance of honesty. Copying } \\
\text { someone's homework is never OK. } \\
\text { Don't worry, you did the right thing. }\end{array}$ \\
\hline
\end{tabular}


VII. Taking into account the last exercise, write the comment according to the situation

\begin{tabular}{|l|l|l|}
\hline Susi0072 & $\begin{array}{l}\text { Comment } 4 \text { Like. Replay Feb 27, 2016 7:10 p.m } \\
\text { I posted some photos of my } 15^{\text {th }} \text { birthday party on } \\
\text { Facebook. One of my friends commented that I looked } \\
\text { skinny and ridiculous in my dress. I feel really hurt. }\end{array}$ & \\
\hline
\end{tabular}

VIII. LISTENING Listen to a presentation about how Colombian citizens deal with emotional intelligence. Describe if the statements are True (T) or false (F)

\begin{tabular}{|l|l|l|}
\hline & T & F \\
\hline a. $80 \%$ don't know what emotional intelligence is. & & \\
\hline b. $90 \%$ try to control their emotions, but can't & & \\
\hline c. 10 people said that they get impatient in stressful situations. & & \\
\hline d. The presenters interviewed their parents for the survey. & & \\
\hline e. One conclusion is that Colombians control their emotions easily. & & \\
\hline
\end{tabular}

IX

what is emotional intelligence?, why it is important to be emotionally intelligent?, then choose two of these incidents and analyze them; finally suggest an advice for solving the situations. Don't forget you can use your cellphone for preparing your oral presentation.

1 I said a bad word to my teacher because she told me to be quiet

2 It is 7:00 p.m. I forgot that today was my best friend's birthday

3 I said something insensitive to friend $A$ about friend $B$. Friend $B$ was listening to me.

4 I laugh at a girl's accent because she is from another region of the country.

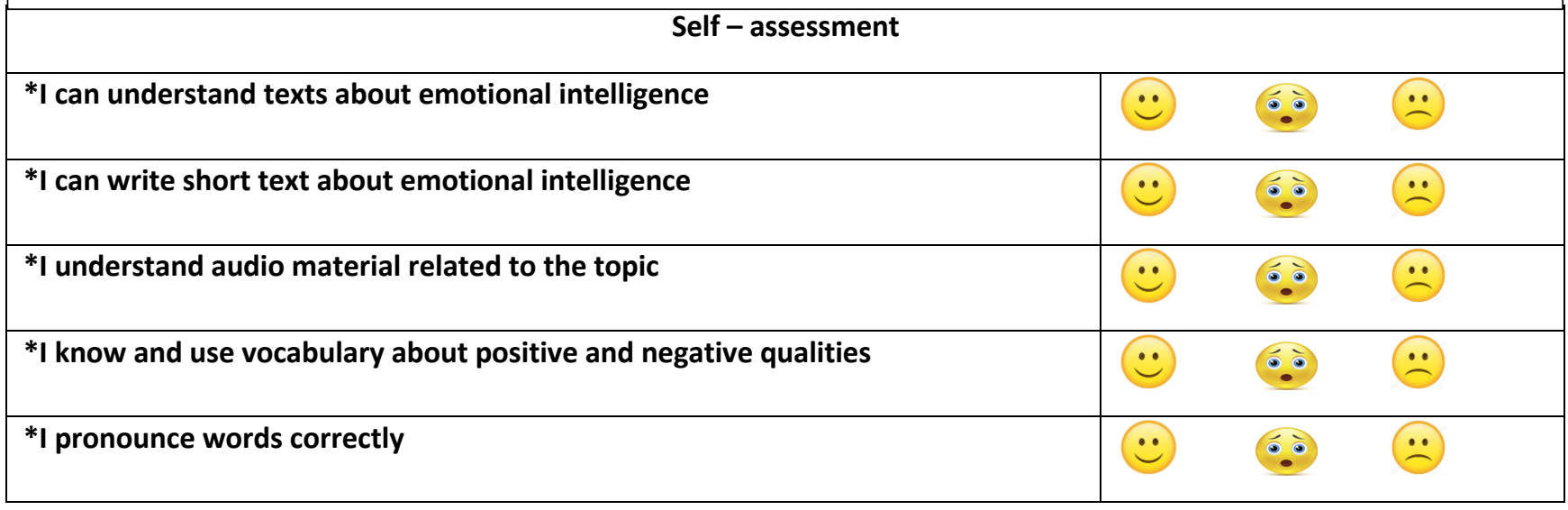




\begin{tabular}{|l|l|}
\hline *I try to do my best effort for the oral report & \\
\hline *My oral performance was & \\
\hline *My oral group's performance was & \\
\hline
\end{tabular}

- Basado en:

- Ministerio de Educación Nacional (2016). Way to go! $8^{\text {th }}$ Grade. Bogotá D.C. - Colombia. Imprensión: www.colombiaaprende.edu.co/colombiabilingue Diseñado por: Yenny A Martín F

Esta obra está bajo una Licencia Creative Commons Attribución-NoCommercial 4.0 International

\section{(cc) EY-NC}

